\title{
Imaginary-Time Matrix Product State Impurity Solver for Dynamical Mean-Field Theory
}

\author{
F. Alexander Wolf, ${ }^{1}$ Ara Go, ${ }^{2}$ Ian P. McCulloch, ${ }^{3}$ Andrew J. Millis, ${ }^{2}$ and Ulrich Schollwöck ${ }^{1,4}$ \\ ${ }^{1}$ Department of Physics, Arnold Sommerfeld Center for Theoretical Physics, \\ LMU Munich, 80333 München, Germany \\ ${ }^{2}$ Department of Physics, Columbia University in the City of New York, New York, New York 10027, USA \\ ${ }^{3}$ Centre for Engineered Quantum Systems, School of Physical Sciences, \\ The University of Queensland, Brisbane, Queensland 4072, Australia \\ ${ }^{4}$ Center for NanoScience, LMU Munich, 80799 München, Germany
}

(Received 31 July 2015; published 24 November 2015)

\begin{abstract}
We present a new impurity solver for dynamical mean-field theory based on imaginary-time evolution of matrix product states. This converges the self-consistency loop on the imaginary-frequency axis and obtains real-frequency information in a final real-time evolution. Relative to computations on the realfrequency axis, required bath sizes are much smaller and no entanglement is generated, so much larger systems can be studied. The power of the method is demonstrated by solutions of a three-band model in the single- and two-site dynamical mean-field approximation. Technical issues are discussed, including details of the method, efficiency as compared to other matrix-product-state-based impurity solvers, bath construction and its relation to real-frequency computations and the analytic continuation problem of quantum Monte Carlo methods, the choice of basis in dynamical cluster approximation, and perspectives for off-diagonal hybridization functions.
\end{abstract}

DOI: 10.1103/PhysRevX.5.041032

\section{INTRODUCTION}

Dynamical mean-field theory (DMFT) in its single-site [1-3] and cluster [4,5] variants is among the most widely employed computational techniques for solving quantum many-body problems. Fundamentally, DMFT in its simplest version becomes exact in the limit of high coordination numbers and was originally applied to simple model Hamiltonians. At least over the past decade, DMFT has been applied increasingly to more realistic models of materials, also of low dimensionality. To this purpose, the initial setup of a single impurity site coupled to a single band has been replaced by impurities consisting of multiple sites and multiple local orbitals. The degree of realism of DMFT is mainly determined by progress made in the size of these generalized impurities.

At the core of a numerical solution of DMFT is an impurity solver: an algorithm for solving a quantum impurity problem. The size of the impurity problem that can be treated is essentially limited by progress in the power of the impurity solver, which emerges as the key limitation of DMFT. The purpose of this paper is to present a new impurity solver and its power to address problems hitherto inaccessible to DMFT. The most prominent

Published by the American Physical Society under the terms of the Creative Commons Attribution 3.0 License. Further distribution of this work must maintain attribution to the author(s) and the published article's title, journal citation, and DOI.
Subject Areas: Computational Physics,

Condensed Matter Physics,

Materials Science

examples of impurity solvers are the continuous-time quantum Monte Carlo (CTQMC) methods [6-8], exact diagonalization (ED) [9-11], the numerical renormalization group (NRG) [12], and the density matrix renormalization group (DMRG) [13]. Other recent suggestions for impurity solvers [14-19], including, in particular, the computationally inexpensive density matrix embedding theory [20], are promising but have not been tested in detail.

While all methods have their strengths, key limitations mean that fundamentally important classes of problems have not yet been adequately addressed. Many of the approaches have been developed in the context of the single-band Hubbard model (one spin-degenerate orbital per site and a correspondingly simple structure for the electron-electron interaction), but in many correlated electron materials of current interest (for example, metallic Fe or $\mathrm{Co}$, or the heavy fermion compounds and the iron arsenide family of high transition temperature superconductors), the physics involves electrons in partly filled $d$ or $f$ shells where multiplet effects associated with orbital degeneracy and its partial lifting by ligand fields and interactions play a crucial role. Our current ability to treat these effects is limited to the single-site approximation and to situations in which the correlated orbitals have a large point group symmetry. Further, intersite correlations beyond the scope of the single-site approximation are believed to play a crucial role in materials with strong electronic anisotropy ("low-dimensional systems," such as the high- $T_{c}$ cuprates, iron arsenide materials, or the 
dicalcogenides). This has been explicitly demonstrated in cluster dynamical mean-field solutions of the twodimensional single-orbital Hubbard model, but the techniques used in this case rely on simplifications associated with the interaction structure of the single-orbital Hubbard model, and no efficient generalization to the multiorbital situation is known. Intersite correlations are believed to be of somewhat less importance for three-dimensional materials, but the question of their importance simply cannot be addressed with current techniques. Thus, in summary, new methods are needed to go beyond (or at least validate) the single-site dynamical mean-field approximation and lift the restriction to the single-orbital or high point symmetry situations.

CTQMC is widely employed, but its application to situations involving low point symmetry, non-Hubbard interactions, or multiple relevant orbitals is limited by the fermionic sign problem. Reaching low temperatures becomes highly computationally expensive while calculating real-frequency information requires analytical continuation, a numerically ill-posed procedure fraught with practical difficulties.

ED makes no assumption on the interaction and does not have a sign problem. It is limited by the size of the Hilbert space that can be studied, meaning in practice that it is restricted to a small number of correlated sites to which only a small number of bath sites can be attached. Recently, improvements have been achieved by considering only restricted subspaces of the Hilbert space [21-24], but the size of the problem remains a significant limitation.

NRG converges the DMFT loop on the real-frequency axis and very effectively obtains real-frequency information in the low-frequency limit. Current applications have been to relatively small problems (the most recent achievement is a solution of the single-site DMFT approximation to a three-band model [25]) and it remains to be seen how far the method can be extended.

DMRG [26] is a set of algorithms operating on the space of matrix product states (MPS) [27]. It has been found to be extremely powerful for the calculation of ground states of one-dimensional quantum systems [27,28]; it was very successfully extended to the calculation of spectral functions which, in contrast to NRG, it obtains with equal resolution across the spectrum (see, e.g., Refs. [29,30]). In pioneering work the method was applied as a DMFT solver by García et al. [13] and Nishimoto et al. [31] with important further work done by these and other authors [32-38]. However, the method has not been widely accepted, perhaps because high-quality data were presented only for the single-site approximation to the single-band Hubbard model. Recently, the method was shown to provide a reliable and highly efficient solver for the two-site dynamical cluster approximation (DCA) approximation to the single-band Hubbard model [39], and insights into the entanglement of the impurity problem make it even more powerful [40]. In view of these advances, DMRG now is a promising candidate for a highly flexible low-cost impurity solver, which can, in addition, be efficiently employed in the nonequilibrium formulation of DMFT [40-42]. However, the key issue of the ability to treat a multiorbital, multisite, low symmetry situation has not yet been demonstrated.

This paper takes a further step forward in the development of DMRG as a tool to study systems with multiple relevant orbitals and important intersite correlations. The important technical advance is a reformulation of the method on the imaginary-time axis. As we show, this strongly reduces entanglement and requires smaller bath sizes, enabling treatment of a large class of problems, including some that are unreachable by other methods, due, e.g., to the sign problem, the size of the correlated cluster, or the number of bands. The price to be paid is a reduced resolution on the real-frequency axis (not needed for converging the DMFT equations or obtaining thermodynamic quantities and phase boundaries), which we study in detail by comparing with calculations that converge the DMFT loop on the real-frequency axis.

The paper is organized as follows. Section II presents the basics of the method. Section III compares the new imaginary-axis formulation with existing real-axis formulations and documents the reduction in bath sizes and the implications for real-axis spectra. We illustrate the method with calculations for three-band models in the single-site and two-site DMFT approximation in Sec. IV and summarize our work in Sec. V. We append discussions of the optimization of typical DMFT Hamiltonians (Appendix A), the entanglement in different representations of the DCA, including a discussion of off-diagonal hybridization functions (Appendix B), and general aspects regarding the computation of Green's functions using MPS (Appendix C).

\section{METHOD}

\section{A. Overview: Green's functions in DMRG}

The computational key challenge in DMFT is the computation of the full frequency dependence of the Green function of a quantum impurity model involving an essentially arbitrary bath. The "size" (number of correlated sites $L_{c}$ ) of the impurity model should be as large as possible and the kinds of interaction that can be treated should be as general as possible. The Green function is used in a self-consistency loop, which may require many iterations for convergence. The solution should be as inexpensive as feasible, and must run automatically, without need for manual optimization of parameters or procedures. In this section, we present a qualitative discussion of the issues involved in computing the Green function using DMRG methods, to motivate the work described in detail below. 
Within DMRG one computes Green's functions by first representing the system ground state $\left|E_{0}\right\rangle$ as a MPS. One then generates a one-electron (one-hole) excitation $\left|\psi_{0}^{>}\right\rangle=$ $d^{\dagger}\left|E_{0}\right\rangle\left(\left|\psi_{0}^{<}\right\rangle=d\left|E_{0}\right\rangle\right)$ by applying a creation (annihilation) operator $d^{\dagger}(d)$ to $\left|E_{0}\right\rangle$. While the state $\left|\psi_{0}^{\gtrless}\right\rangle$ is at most as entangled as the ground state $\left|E_{0}\right\rangle[30]$, in order to compute a Green function one has to perform further operations on $\left|\psi_{0}^{\gtrless}\right\rangle$. These operations typically increase entanglement and by that the bond dimension of a MPS, which ultimately limits all computations.

Let us be more concrete and consider a general MPS of bond dimension $m$ for a system with $L$ sites and open boundary conditions. Defining $A^{\sigma_{i}}, B^{\sigma_{i}} \in \mathbb{C}^{m \times m}$ for $i \neq 1$, $L$ and $A^{\sigma_{1}} \in \mathbb{C}^{1 \times m}, B^{\sigma_{L}} \in \mathbb{C}^{m \times 1}$, where $\sigma_{i} \in\{0, \uparrow, \downarrow, \uparrow \downarrow\}$ labels a local basis state of the Hilbert space, any MPS can be represented as [27]

$\left|\psi_{\mathrm{MPS}}\right\rangle=\sum_{\sigma_{1}, \ldots, \sigma_{L}} A^{\sigma_{1}} \ldots A^{\sigma_{l}} S B^{\sigma_{l+1}} \ldots B^{\sigma_{L}}\left|\sigma_{1}, \ldots, \sigma_{L}\right\rangle$,

where $S=\operatorname{diag}\left(s_{1}, \ldots, s_{m}\right)$ is a diagonal matrix and $A^{\sigma_{i}}$ are left normalized and $B^{\sigma_{i}}$ are right normalized, respectively:

$$
\sum_{\sigma_{i}} A^{\sigma_{i}^{\dagger} \dagger} A^{\sigma_{i}}=I, \quad \sum_{\sigma_{i}} B^{\sigma_{i}} B^{\sigma_{i}^{\dagger}}=I .
$$

Here, $I$ are identity matrices. Left and right normalization make Eq. (1) the Schmidt decomposition of $\left|\psi_{\text {MPS }}\right\rangle$ that is associated with partitioning the system at bond $(i, i+1)$. The bond entanglement entropy for the associated reduced density matrix can therefore simply be read off from Eq. (1) [27]:

$$
S_{(i, i+1)}^{\mathrm{ent}}=\sum_{\nu=1}^{m} s_{\nu}^{2} \ln s_{\nu}^{2}
$$

When subsequently we refer to an entanglement growth associated with repeated operations on $\left|\psi_{\text {MPS }}\right\rangle$, this implies the need to adjust the bond dimension $m$ such that $\left|\psi_{\text {MPS }}\right\rangle$ still faithfully represents a physical state. If entanglement in the physical state becomes too large, we have to choose $m$ so large that computations with MPS become impractical.

Since the first suggestion for computing spectral functions within DMRG [43], the field has evolved by the important development of the correction vector method $[44,45]$. The subsequent understanding of the connection between DMRG and MPS [27] opened the door to many further approaches to computing spectral and Green's functions, in particular, time evolution and subsequent Fourier transform [46,47], an improved Lanczos algorithm [48], and the Chebyshev recursion [29,30,49]. All of these are formulated for the calculation of spectral functions at $T=0$, as considered in the present paper, and came at much cheaper computational cost than the correction vector method [29,30]. We note that for $T>0$, there are perspectives for even more powerful algorithms: it was recently demonstrated that the numerically exact spectral function of a molecule consisting of several hundreds of interacting spins could be computed [50].

These developments (see Appendix C for more details) make MPS-based solvers an attractive possibility for dynamical mean-field theory. However, the growth of entanglement arising in all calculations of the Green function has limited the system sizes that have been addressed to date. Also, in MPS computations manual adjustments, for example, choosing optimal broadening [29] or combining results of different systems sizes [48], are still common practice. In the rest of this section, we show that these problems can, to a large degree, be circumvented by computing Matsubara Green's functions using imaginary-time evolution. The imaginary-time framework naturally extends existing techniques based on real-time evolution $[38,40]$, which have been shown to provide the currently most efficient algorithmic approach to compute real-frequency spectral functions [30].

\section{B. Imaginary-time computation}

The central objects of technical interest in this paper are the greater and the lesser correlation functions $\tilde{G}^{\gtrless}$, which we define for imaginary time $\tau$ :

$$
\begin{aligned}
\tilde{G}^{\gtrless}(\tau) & =\left\langle\psi_{0}^{\gtrless}\left|e^{\mp\left(H-E_{0}\right) \tau}\right| \psi_{0}^{\gtrless}\right\rangle, \\
\tilde{G}^{\gtrless}(i t) & =\left\langle\psi_{0}^{\gtrless}\left|e^{\mp i\left(H-E_{0}\right) t}\right| \psi_{0}^{\gtrless}\right\rangle .
\end{aligned}
$$

In the second line, we evaluate $\left.\tilde{G}^{\gtrless}(\tau)\right|_{\tau=i t}$ and by that obtain a correlation function for real time $t$, which will be useful later on. The functions $\tilde{G}^{\gtrless}$ carry spin and orbital indices associated with the spin and orbital indices of the single-particle (hole) excitation $\left|\psi_{0}^{\gtrless}\right\rangle$, but these indices are not explicitly written here. We discuss the relationship of $\tilde{G}^{\gtrless}$ to the physical Green's functions (which we denote by $G$ ) below.

While it is not essential in principle, we evaluate Eq. (4) using a Krylov algorithm [51], which represents the timeevolution operator in a local Krylov space and is able to treat Hamiltonians with long-ranged interactions. Before performing a time-evolution computation, one has to compute the initial state $\left|\psi_{0}^{\gtrless}\right\rangle$ using a MPS optimization of the ground state. As impurity models come with open boundary conditions, this is well suited for DMRG. We discuss this optimization for typical DMFT Hamiltonians in Appendix A 1.

Figure 1 presents representative results based on parameters obtained from a two-site DMFT solution of the Hubbard model. Figure 1(a) shows the time evolution of $\tilde{G}^{\gtrless}(\tau)$ out to times as long as 350 times the basic time scale (inverse half-bandwidth $D$ ) of the model, which suffices to 

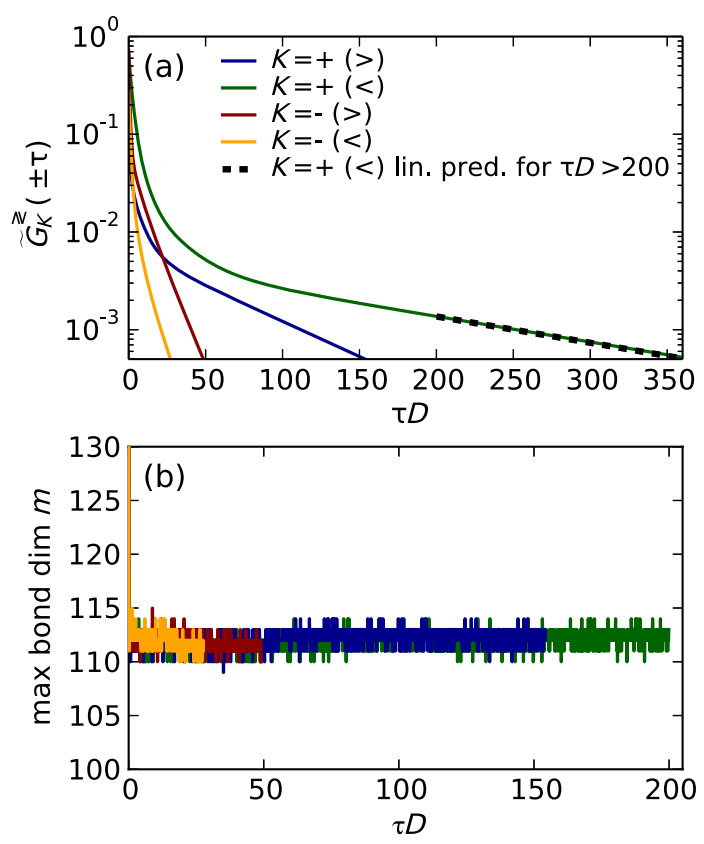

FIG. 1. (a) Imaginary-time correlation functions $\tilde{G}^{\gtrless}(\tau)$ defined in Eq. (4a) for an impurity model arising in the context of the twosite dynamical cluster approximation to the single-band Hubbard model on the square lattice with next-nearest neighbor hopping $t^{\prime} / t=0.3$, half-bandwidth $D=4 t$, interaction $U=2.5 D$, and band filling $n=0.96$ in the paramagnetic phase. See Ref. [52] for definition of the model and the meaning of the orbital (patch) quantum number $K= \pm$. The dashed line is obtained using linear prediction for times $\tau D \geq 200$. (b) Maximal bond dimension $m$ of time-evolved states. The MPS computation uses a Hamiltonian representation of the discrete (approximate) impurity model with $L_{c}=2$ correlated sites and $L_{b}=14$ bath sites. The hybridization function of the impurity model $\Lambda^{\text {discr }}$ is fitted using Eq. (13) for $\beta_{\text {eff }}=315 / D$ and $\alpha=0$. For the ground state optimization, we enforce a maximal bond dimension of $m=300$. The Krylov time evolution uses a time step of $\Delta t=0.1 / D$ and allows for a maximal global truncation error of $10^{-4}$ at each time step, adjusting bond dimensions automatically. This leads to an immediate decay of $m$ at $\tau \simeq 0$ from $m=300$ down to $m \simeq 110$, as seen in (b). We use the global SU(2) symmetry of the Hamiltonian to reach these low values of the bond dimension.

converge $\tilde{G}^{\gtrless}(\tau)$ to a precision of $5 \times 10^{-4}$. Figure $1(\mathrm{~b})$ demonstrates the key advantage that makes this computation possible: the lack of growth of maximal bond dimensions $m$ with time of the associated imaginary-time evolved states $\left|\psi^{\gtrless}(\tau)\right\rangle=e^{-\left(H-E_{0}\right) \tau}\left|\psi_{0} \gtrless\right\rangle$. The imaginary-time evolution operator does not create entanglement as it projects on the lowly entangled ground state.

Figure 1(a) reveals additional information about the nature and rate of convergence of $\tilde{G}^{\gtrless}(\tau)$. In the insulating phase, $H$ has a gap and $\tilde{G}^{\gtrless}(\tau)$ decays exponentially irrespective of whether one considers a finite system or the thermodynamic limit. In the metallic phase, $\tilde{G}^{\gtrless}(\tau)$ decays algebraically in the thermodynamic limit. For a finite system though, there always remains a small gap, and even though the decay resembles an algebraic decay for short times, it always becomes exponential at long times. The exponential decay can be exploited to speed up computations considerably by a simple technique known as linear prediction [30,47,53,54]. This technique is an efficient formulation of the fitting problem for the ansatz function $f(\tau)=\sum_{n} \alpha_{n} e^{\beta_{n} \tau}, \alpha_{n}, \beta_{n} \in \mathbb{C}, \tau \in \mathbb{R}$, which can then be used to reliably extrapolate functions with an exponentially decaying envelope. This is illustrated by the dashed black line in Fig. 1(a), which is fitted to match $\tilde{G}^{\gtrless}(\tau)$ for $\tau D \in[150,200]$ and is then extrapolated for higher times. The solid green line, by contrast, is the result of the MPS computation. Agreement can be seen to be perfect.

\section{Physical Green's functions}

Of particular interest in the rest of this paper are the imaginary-time Green functions $G^{\text {mat }}(\tau)$ defined via

$$
G^{\mathrm{mat}}(\tau)=-\theta(\tau) \tilde{G}^{>}(\tau)+\theta(-\tau) \tilde{G}^{<}(\tau),
$$

whose Fourier transform gives the Matsubara Green function [we distinguish the Fourier transform $G^{\text {mat }}\left(i \omega_{n}\right)$ of $G^{\text {mat }}(\tau)$ only by its argument to keep the notation simple]

$$
G^{\mathrm{mat}}\left(i \omega_{n}\right)=\int_{-\infty}^{\infty} d \tau e^{i \omega_{n} \tau} G^{\mathrm{mat}}(\tau),
$$

at zero temperature, where $\omega_{n}=(2 n+1) \pi / \beta$ and $\beta \rightarrow \infty$. We are also interested in the retarded real-time Green function,

$$
G^{\mathrm{ret}}(t)=-i \theta(t)\left[\tilde{G}^{>}(i t)+\tilde{G}^{<}(i t)\right],
$$

from which the retarded frequency-dependent Green function is obtained as [again, we distinguish the Fourier transform $G^{\text {ret }}(\omega)$ of $G^{\text {ret }}(t)$ only by its argument]

$$
G^{\mathrm{ret}}(\omega)=\int_{-\infty}^{\infty} d t e^{i\left(\omega+i 0^{+}\right) t} G^{\mathrm{ret}}(t) .
$$

This allows one to obtain the spectral function as $A(\omega)=-(1 / \pi) \operatorname{Im} G^{\text {ret }}(\omega)$.

In numerical practice, we evaluate the Fourier transforms leading to Eqs. (6) and (8) approximately as

$$
\begin{aligned}
G^{\operatorname{mat}}\left(i \omega_{n}\right) & =-\int_{0}^{\tau_{\max }} d \tau \tilde{G}^{>}(\tau) e^{i \omega_{n} t}+\int_{-\tau_{\max }}^{0} d \tau \tilde{G}^{<}(\tau) e^{i \omega_{n} t}, \\
G^{\mathrm{ret}}(\omega) & =-i \int_{0}^{t_{\max }} d t\left[\tilde{G}^{>}(i t)+\tilde{G}^{<}(i t)\right] e^{i \omega t},
\end{aligned}
$$

with cutoff times $\tau_{\max }$ and $t_{\max }$. This approximation is controlled only if we are able to reach long enough times 
$\tau_{\max }$ and $t_{\max }$, such that $\tilde{G}^{\gtrless}(\tau)$ and $\tilde{G}^{\gtrless}(i t)$ have converged to zero to any desired accuracy.

In contrast to a computation on the imaginary axis, reaching arbitrarily long times $t_{\max }$ on the real axis is prohibited by a logarithmic growth of entanglement, which comes with a power-law growth of bond dimensions. In addition, finite-size effects are a severe source of errors because the long-time behavior is determined by the bath size. For a numerically exact computation, one has to choose the system large enough to observe exponential "pseudoconvergence" of $\tilde{G}^{\gtrless}(i t)$ to zero [30]. This means that-after an initial regime-the envelope of $\tilde{G}^{\gtrless}(i t)$ decays exponentially up to the time at which finite-size effects begin to be resolved. In the context of the present paper, we deal with small system sizes and will never observe pseudoconvergence. In particular, there is no exponential pseudoconvergence, so that linear prediction cannot be employed [30]. Therefore, when computing the real-frequency spectral function after converging the DMFT loop, one has to use the further approximation of damping the finite-size effects that emerge at long times by computing, instead of $G^{\text {ret }}(\omega)$ in Eq. (9),

$$
G_{\eta}^{\mathrm{ret}}(\omega)=-i \int_{0}^{\infty} d t\left[\tilde{G}^{>}(i t)+\tilde{G}^{<}(i t)\right] e^{i \omega t} e^{-\eta^{2} t^{2} / 2},
$$

which yields the broadened spectral function $A_{\eta}(\omega)=$ $-(1 / \pi) \operatorname{Im} G_{\eta}^{\mathrm{ret}}(\omega)=(1 / \sqrt{2 \pi} \eta) \int d \omega^{\prime} A\left(\omega^{\prime}\right) e^{-\left(\omega-\omega^{\prime}\right)^{2} / 2 \eta^{2}}$. Instead of a Gaussian damping and broadening, one could also use an exponential damping leading to Lorentzian broadening, which damps out the original time-evolution information more strongly, though.

Before presenting detailed benchmark results for the solution of DMFT using imaginary-time evolution of MPS, let us clarify the price we have to pay for profiting from the great advantage of not facing entanglement growth. We do this by comparing the imaginary-time approach (ITMPS) to approaches that solve the DMFT loop on the real axis.

\section{COMPARISON OF IMAGINARY-AXIS WITH REAL-AXIS COMPUTATIONS}

The self-consistency equation in DMFT relates an impurity model specified by a hybridization function and a self-energy to a lattice model specified by a lattice Hamiltonian and the same self-energy. We discuss the issues using the example of the dynamical cluster approximation to the single-band Hubbard model:

$$
\begin{aligned}
G_{K}^{\mathrm{latt}}(z) & =\frac{N_{c}}{N} \sum_{k \in \mathcal{P}_{K}} \frac{1}{z+\mu-\varepsilon_{k}-\Sigma_{K}(z)}, \\
& \stackrel{!}{=}\left[z+\mu-\varepsilon_{K}-\Sigma_{K}(z)-\Lambda_{K}(z)\right]^{-1} \\
& =G_{K}^{\mathrm{imp}}(z) .
\end{aligned}
$$

Here, $\varepsilon_{k}$ denotes the single-particle dispersion of the lattice and $\mu$ is the chemical potential. In the dynamical cluster approximation, the Brillouin zone, consisting in $N$ momentum vectors $k$, is covered by $N_{c}$ (for single band $L_{c}=N_{c}$ ) equal-area tiles (patches), labeled here by $P_{K}$ and the selfenergy $\Sigma_{K}(\omega)$ is taken to be piecewise constant, with $\Sigma_{K}(\omega)$ being a potentially different function of frequency in each tile. The impurity model is specified by the on-site energy $\varepsilon_{K}$ and the hybridization function $\Lambda_{K}(z)$, which is to be determined using a fixed point iteration referred to as the DMFT loop. This works as follows. Make an initial guess for $\Lambda_{K}(z)$, then compute $\Sigma_{K}(z)$ using a MPS calculation of $G_{K}^{\text {imp }}(z)$ via

$$
\Sigma_{K}(z)=z+\mu-\varepsilon_{K}-\Lambda_{K}(z)-\left[G_{K}^{\mathrm{imp}}(z)\right]^{-1},
$$

then update $\Lambda_{K}$ using the first line of Eq. (11) via

$$
\Lambda_{K}(z)=z+\mu-\varepsilon_{K}-\Sigma_{K}(z)-\left[G_{K}^{\text {latt }}(z)\right]^{-1},
$$

and repeat this procedure until convergence.

We discuss two aspects of the comparison of realand imaginary-frequency solutions of the DMFT selfconsistency equation (11). The first has to do with the number of bath sites needed to obtain a solution of the selfconsistency equation. The second is the accuracy to which the spectral functions of physical interest can be reproduced.

The DMFT self-consistency equation (11) defines the hybridization function $\Lambda_{K}$ as a continuous function in terms of the difference between the computed self-energy and the inverse of the lattice Green function. In DMRG-type methods, the hybridization function $\Lambda_{K}$ is approximated as the hybridization function $\Lambda_{K}^{\mathrm{discr}}$ (a sum of poles) of a discrete impurity model with a finite number $L_{b}$ of bath sites. If the number $L_{b}$ of bath sites is too small, one cannot construct a meaningful approximation on the real axis [55] and a DMFT loop cannot be converged. For this reason, DMRG-based solutions of DMFT up to now [13,31-40], all of which were real axis computations, have been performed using numbers of bath sites of at least $L_{b} / L_{c} \gtrsim 30$, and in the case of the single-band Hubbard model, even much more, $L_{b} / L_{c} \gtrsim 120$. Use of such a large number of bath sites means that with modest broadening the hybridization function can be reasonably approximated as a continuum, enabling a stable solution of Eq. (11).

By contrast, formulating the problem on the imaginary axis (as is typically done in standard ED solvers where the number of bath sites is strictly limited) automatically smoothens the hybridization function $\Lambda_{K}^{\text {discr }}$ and permits a stable solution. From the imaginary-axis solution, one must then determine the discrete set of bath parameters to represent $\Lambda_{K}^{\text {discr }}$. This is typically done $[9,11,56]$ by numerical minimization of a cost function defined as 


$$
\chi^{2}=\frac{1}{N_{\text {fit }}} \sum_{n=1}^{N_{\text {fit }}} \omega_{n}^{-\alpha}\left|\Lambda_{K}\left(i \omega_{n}\right)-\Lambda_{K}^{\operatorname{discr}}\left(i \omega_{n}\right)\right|^{2}
$$

Here, $\alpha$ defines a weighting function $\omega_{n}^{-\alpha}$. Choosing $\alpha>0$, e.g., $\alpha=1$, attributes more weight to smaller frequencies $[11,56,57]$, which we find helpful when using small bath sizes $L_{b} / L_{c}<5$. To define the frequency grid for the fit $\omega_{n}=(2 n+1) \pi / \beta_{\text {eff }}$, one defines a fictitious inverse temperature $\beta_{\text {eff }}$, which has no physical significance. We further employ a cutoff frequency $\omega_{c}$, which implies a finite number $N_{\text {fit }}$ of fitted Matsubara frequencies.

If one tries to define an analogous cost function for the real axis, the result is useless as then $\Lambda_{K}^{\operatorname{discr}}\left(\omega+i 0^{+}\right)$is a sum of poles, whereas the hybridization function $\Lambda_{K}\left(\omega+i 0^{+}\right)$, as encountered in Eq. (11), is continuous [55]. One can overcome this problem only when using a Lindbladt formalism [58], which increases the complexity of the problem substantially.

The minimization of Eq. (13) is done using standard numerical optimization. The optimization in the initial DMFT iteration should be done using a global optimization scheme [59], and in subsequent iterations using a local optimization scheme (e.g., conjugate gradient), which takes as an initial guess for the new bath parameters the values of the previous iteration. Figure 2 shows the convergence of the fit of the hybridization function with the number of bath sites $L_{b} / L_{c}$. For $L_{b} / L_{c}=7$, one already obtains errors as little as $\simeq 10^{-3}$, and for values $L_{b} / L_{c} \gtrsim 9$, the quality of the fit already stops improving. It is at this point, where we (and all ED-like techniques) face the problem of "analytic continuation" encountered in imaginary-time CTQMC methods, namely, that Green's functions on the imaginary axis encode information in a much less usable form than on the real axis.

Consider again the example of the two-site DCA for the single-band Hubbard model on the square lattice. In Ref. [39], this problem has been solved entirely on the

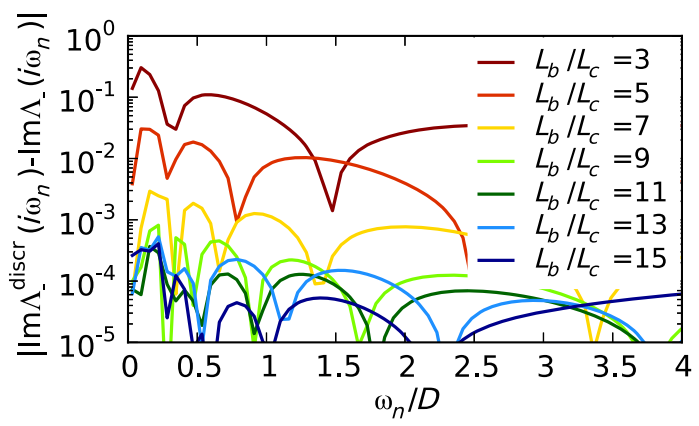

FIG. 2. Fit of the hybridization function in the two-site DCA problem studied in Figs. 1 and 3, but here for the case $U=0$. The minimization [Eq. (13)] is done using $\alpha=0$ and a frequency grid defined by $\beta_{\text {eff }}=100 / D$ and a cutoff frequency of $\omega_{c}=6 D$. Evidently, the quality of the fit does not improve any more for $L_{b} / L_{c} \gtrsim 9$. real axis using $L_{b} / L_{c}=39$ bath orbitals. Here, we converge the DMFT loop on the imaginary axis and compute the spectral function in a final real-time evolution using $L_{b} / L_{c}=3,5,7$ bath orbitals. We compare both solutions
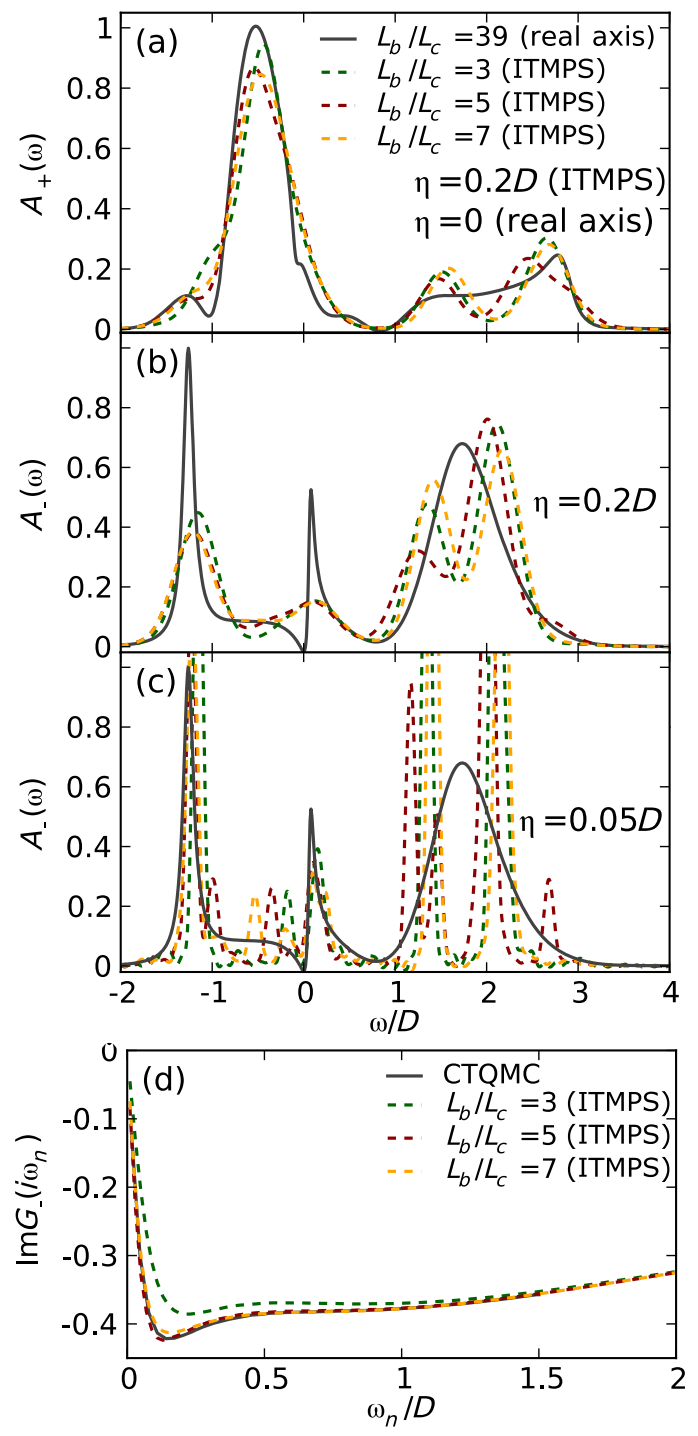

FIG. 3. Real- and imaginary-frequency Green's functions computed by converging the DMFT self-consistency equation [Eq. (11)] for the two-site dynamical cluster approximation to the single-band Hubbard model on the square lattice with nextnearest neighbor hopping $t^{\prime} / t=0.3$, half-bandwidth $D=4 t$, interaction $U=2.5 \mathrm{D}$, and band filling $n=0.96$ in the paramagnetic phase (as in Fig. 1). See Ref. [52] for definition of the model and the meaning of the orbital (patch) quantum number $K= \pm$. (a)-(c) Electron spectral function $A_{+}(\omega)$ (a) and $A_{-}(\omega)$ (b) obtained by converging on imaginary-frequency axis (ITMPS) using number of bath sites and different broadenings as specified in the figure, and compared to unbroadened $(\eta=0)$ real-frequency axis computation using $L_{b} / L_{c}=39$ bath sites per correlated site of Ref. [39]. (d) Converged Matsubara Green's function for number of bath sites shown, compared to numerically exact quantum Monte Carlo result of Ref. [52], computed at $\beta=200 / D$. 
in Fig. 3(a). Whereas for the (central) momentum patch "+" shown in Fig. 3(a), we find satisfactory agreement of the imaginary-axis with the real-axis calculation, this is not the case for the (outer) momentum patch "-" shown in Fig. 3(b), even though the corresponding imaginary-axis Green function is well reproduced; see Fig. 3(d). Evidently, in Fig. 3(b), the central peak and the pseudogap at the Fermi edge are smeared out by a broadening $\eta=0.2 D$ that hides finite-size effects to a large degree. Reducing the broadening to $\eta=0.05 D$, as shown in Fig. 3(c), again reveals the pseudogap and the central peak, but together with unphysical finite-size effects. We observe that the nature of these finite-size effects is qualitatively comparable when using different numbers of bath sites, $L_{b} / L_{c}=3,5,7$. On the imaginary axis, by contrast, $L_{b} / L_{c}=5,7$ still improve over $L_{b} / L_{c}=3$ and almost agree with the numerically exact QMC data for $\beta=200 / D$ of Ref. [52]; see Fig. 3(d). However, we emphasize that even with the modest number of bath sites used here, the basic features of the spectral function are reproduced (for example, the areas in given frequency ranges).

\section{THREE-BAND CALCULATIONS}

\section{A. Three-band model in single-site DMFT}

We now demonstrate the power of the method by applying it to three-band problems in the single-site approximation (where comparison to existing calculations can be made) and the two-site approximation. Both have hitherto not been accessible to DMFT + DMRG computations.

We study the three-band Hubbard-Kanamori model with Hamiltonian (omitting the site index $i$ in the following definition of $H_{\mathrm{loc}, i}$ )

$$
\begin{aligned}
H= & \sum_{k, a, b, \sigma} \varepsilon_{k}^{a b} d_{k, a, \sigma}^{\dagger} d_{k, b, \sigma}+\sum_{i} H_{\mathrm{loc}, i}, \\
H_{\mathrm{loc}}= & -\sum_{a, \sigma}\left(\mu-\Delta_{a}\right) n_{a, \sigma}+\sum_{a} U n_{a, \uparrow} n_{a, \downarrow} \\
& +\sum_{a>b, \sigma}\left[U^{\prime} n_{a, \sigma} n_{b,-\sigma}+\left(U^{\prime}-J\right) n_{a, \sigma} n_{b, \sigma}\right] \\
& -\sum_{a \neq b} J\left(d_{a, \downarrow}^{\dagger} d_{b, \uparrow}^{\dagger} d_{b, \downarrow} d_{a, \uparrow}+d_{b, \uparrow}^{\dagger} d_{b, \downarrow}^{\dagger} d_{a, \uparrow} d_{a, \downarrow}+\text { H.c. }\right),
\end{aligned}
$$

where $i$ labels sites in a lattice and $k$ labels wave vectors in the first Brillouin zone, $n_{i, a, \sigma}=d_{i, a, \sigma}^{\dagger} d_{i, a, \sigma}$ is the density of electrons of spin $\sigma$ in orbital $a$ on site $i, \mu$ is the chemical potential, $\Delta_{a}$ is a level shift for orbital $a, \varepsilon_{k}^{a b}$ is the band dispersion, $U$ is the intraorbital and $U^{\prime}$ the interorbital Coulomb interaction, and $J$ is the coefficient of the Hund coupling and pair-hopping terms. We adopt the conventional choice of parameters, $U^{\prime}=U-2 J$, which follows from symmetry considerations for $d$ orbitals in free space and holds (at least for reasonably symmetric situations) for the $t_{2 g}$ manifold in solids [60].

We study the orbital-diagonal and orbital-degenerate case $\left(\Delta_{a}=0\right)$ on the Bethe lattice, i.e., the noninteracting density of states is semielliptic,

$$
A_{a, 0}(\omega)=\frac{1}{\pi t} \sqrt{1-\left(\frac{\omega}{2 t}\right)^{2}} .
$$

In the single-site approximation, the impurity Hamiltonian used within DMFT is given by

$$
\begin{aligned}
H & =H_{\mathrm{loc}}+H_{\mathrm{coupl}}+H_{\mathrm{bath}}, \\
H_{\text {coupl }} & =\sum_{l, a, \sigma} V_{l, a, \sigma} d_{a, \sigma}^{\dagger} c_{l, a, \sigma}+\text { H.c., } \\
H_{\text {bath }} & =\sum_{l, a, \sigma} \varepsilon_{l, a . \sigma} c_{l, a, \sigma}^{\dagger} c_{l, a, \sigma},
\end{aligned}
$$

where $c_{l, a, \sigma}^{\dagger}$ creates a fermion in the bath orbital $l, V_{l, a, \sigma}$ describes the coupling of the impurity to the orbital $l$, and $\varepsilon_{l, a . \sigma}$ denotes the potential energy of orbital $l$. The hybridization function is then given by

$$
\Lambda_{a, \sigma}^{\mathrm{discr}}(z)=\sum_{l=1}^{L_{b} / L_{c}} \frac{\left|V_{l, a, \sigma}\right|^{2}}{z-\varepsilon_{l, a . \sigma}} .
$$

Figure 4 compares the dependence of the particle density $n$ on the chemical potential $\mu$ obtained by the MPS methods used here to those obtained by numerically exact CTQMC methods [61]. The plateaus in $n(\mu)$ are the Mott insulating regimes of the phase diagram. The agreement is very good in general, confirming the reliability of our new procedure even with only three bath sites per correlated site. This leads to an extremely cheap computation, for which a single iteration of the DMFT loop takes about $30 \mathrm{~min}$ on two 2.8- GHz cores (see Appendix A 2 for more details).

In Fig. 5(a), we show a more stringent test, namely, the dependence of the self-energy on Matsubara frequency, in a parameter regime where the self-energy was previously found [62] to exhibit an anomalous $\omega^{1 / 2}$ frequency dependence and (in some regimes) a nonzero intercept as $\omega \rightarrow 0$. These phenomena are associated with a spinfreezing transition $[61,62]$.

Figure 5(a) shows that the low-frequency $\omega \lesssim t$ selfenergy obtained using CTQMC is already accurately reproduced even for the computationally inexpensive choice of $L_{b} / L_{c}=3$, although one observes deviations for the high-frequency behavior. The deviations at high frequency decrease as the number of bath sites is increased, although full convergence at all frequencies has not been demonstrated. Figure 5(b) shows that the deviations are linked to the impossibility of fitting the hybridization function equally well for all frequencies using only a small 


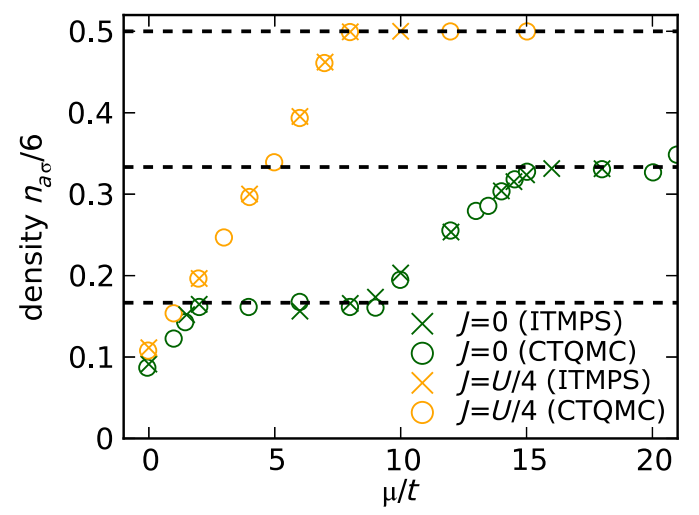

FIG. 4. Density per orbital as function of chemical potential for three-band Hubbard-Kanamori model Eq. (14) using the semielliptic density of states Eq. (15) and $U=12 t$, obtained from single-site DMFT approximation evaluated using imaginary-time MPS (crosses) and CTQMC data (circles, Fig. 1 of Ref. [61], inverse temperature $\beta=50 / t$ ). In the DMRG computations the bath fitting is performed using $\beta_{\text {eff }}=100 / t, \omega_{c}=6 t$, and $\alpha=1$, with three bath sites per correlated site $\left(L_{b} / L_{c}=3\right)$. The maximal matrix dimensions is $m=300$ for the ground state calculation, exploiting the SU(2) symmetry, which leads to the high precision $\left\langle(H-E)^{2}\right\rangle \simeq 10^{-14}$. For the time evolution, we compute $\tilde{G}_{a}^{\gtrless}(\tau)$ in Eq. (4a) in steps of $\Delta \tau=0.1 / t$ allowing for a global truncation error of $5 \times 10^{-4}$ per step, up to imaginary time $\tau_{\max }=100 / t$, and use linear prediction for higher times.

number of bath sites. The large deviations at high frequencies are due to the choice $\alpha=1$ in Eq. (13), which enforces good agreement for low frequencies and allows us to successfully reproduce the metal-insulator transition (MIT) in Fig. 4. Increasing the number of bath sites to $L_{b} / L_{c}=5$ leads to a much better approximation of the hybridization function also for high frequencies, with concomitant improvement in the self-energy [Fig. 5(a)].

\section{B. Three-band model in two-site DCA}

We now present results obtained using a two-site DCA approximation to the three-band model of the previous section. For this problem there are no low-temperature results available in the literature. The size of the problem is beyond the scope of standard ED. The truncated configuration interaction $(\mathrm{CI})$ impurity solver [20] allows one to access a relatively high number of bath sites but is limited in the number of correlated sites: for example, in Ref. [24], a problem with $L_{c}=3$ and $L_{b}=30$ was computed, and in Ref. [56], one with $L_{c}=4$ and $L_{b}=20$. The three-band two-site DCA though has $L_{c}=6$ correlated sites and it remains to be seen whether this is in reach for the CI solver. The problem is also challenging for standard CTQMC. Recent technical improvements on mitigating the sign problem [63] enabled Ref. [64] to treat this model at the temperature of $T=0.025 D$, with $D$ the half-bandwidth, although large computational resources were required. As the authors noted, this temperature is high relative to the
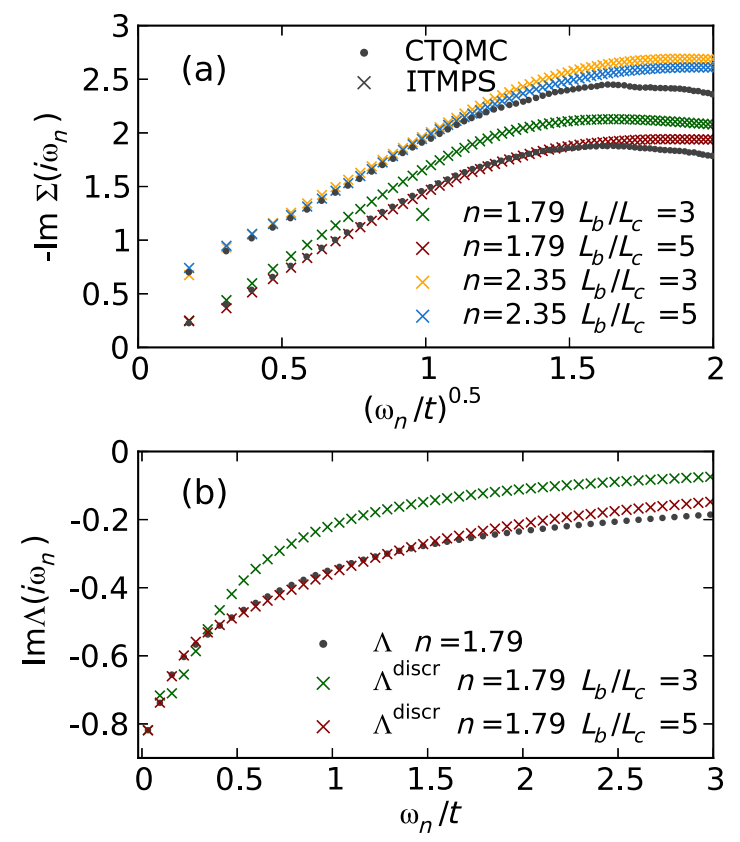

FIG. 5. (a) Imaginary part of Matsubara axis self-energy $\Sigma$ and (b) imaginary part of hybridization function $\Lambda$ for densities shown obtained from converged ITMPS solution of single-site DMFT for three-band Hubbard-Kanamori model [Eq. (16)] for $U=8 t$ and $J=U / 6$. Crosses represent ITMPS data and black circles depict CTQMC data from Fig. 3 of Ref. [62], computed at inverse temperature $\beta=100 / t$. We choose all parameters as described in the caption of Fig. 4; in particular, for the bath fitting [Eq. (13)], we use $\beta_{\text {eff }}=100 / t, \omega_{c}=6 t$, and $\alpha=1$. Choosing $\alpha=1$ enforces agreement for low frequencies at the price of disagreement at high frequencies, which is observed in both (a) and (b). In (b), $\Lambda$ denotes the hybridization function that is fitted with the hybridization function $\Lambda^{\text {discr }}$ of the discrete impurity model.

effective Fermi energy. In a study of a simpler two-band two-site model the authors reached $T=0.0125 D$, but this is not yet low enough to resolve a Fermi liquid phase (if one exists for these parameters) [63].

We study the model on the two-dimensional square lattice, i.e., using $\varepsilon_{k}^{a b}=-2 t\left(\cos k_{x}+\cos k_{y}\right) \delta^{a b}$. We use the momentum patching of Ref. [52]; this definition is also used in the single-band computations of Figs. 1 and 3. We note that this model is not directly relevant to layered materials where the $t_{2 g}$ orbitals are relevant, because in the physical situation the two dimensionality will break the threefold orbital degeneracy. However the system is well defined as a theoretical model and is useful to demonstrate the power of our methods.

As is the case for the CI method, the DMRG method we use here is easily able to treat a large number of bath sites if the number of correlated sites is small: for $L_{c}=1$, DMRG has already often be proven to treat $L_{b}>120$ bath sites, and for $L_{c}=2, L_{b}>80$ is easily accessible [39,40]. However, for more correlated sites, the number of bath 
sites that can be added at given computational cost decreases. For $L_{c}=6$, we use $L_{b}=18$, i.e., $L_{b} / L_{c}=3$, which we showed to be sufficient to produce reliable results (previous sections). The solution does not require overly large computation resources (computation time of several hours per DMFT iteration on two cores).

We test the two-site calculation by converging the DMFT loop for the three-band Hubbard model Eq. (16) with $U^{\prime}$ and $J=0$ and comparing the results with a corresponding two-site single-band DCA. Perfect agreement is obtained (not shown). Nonzero values of $U^{\prime}$ and $J$ create additional entanglement and make computations more costly. It is then a decisive question whether a real-space or a momentum-space representation of the impurity cluster is less entangled. We discuss this in Appendix B, finding that for the single-band Hubbard interaction both representations yield similar entanglement, whereas for the HubbardKanamori interaction, the real-space representation is much less entangled. Computational cost is therefore tremendously reduced by using the real-space representation, which comes with an off-diagonal hybridization function. This is the opposite behavior as observed for the QMC method, where the off-diagonal hybridization function creates a severe sign problem. We further note that in the real-space representation, strong interactions yield a less and less entangled impurity problem, as electrons become more and more localized.

We now present results for the more physically relevant case, $U^{\prime}=U-2 J$ with $J=U / 4$. For these parameters, at half filling the critical interaction for the MIT in the singlesite DMFT approximation is $U_{c} \simeq 1.3 D$ [65]. Figure 6(a) shows that our results are consistent with this estimate: the dashed lines depict the single-site $(1 s)$ results, showing a metallic solution (spectral function nonzero at $\omega=0$ ) for $U=D$, and an insulating solution (spectral function zero at $\omega=0$ ) for $U=2 D$. In the two-site (2s) DCA (solid lines), by contrast, the critical value $U_{c}$ for the MIT is lowered. Even at $U=D$, the $\omega=0$ spectral function is zero [the small nonzero value in Fig. 6(a) is an effect of broadening, as seen in Fig. 6(b)]. The different nature of the metallic and insulating solutions is also visible on the imaginary axis in the different nature of the decay of the imaginary-time Green function. This is plotted in Fig. 6(c) for $U=D$; clearly, a power-law decay is observed for the metallic solution obtained in the single-site DMFT, whereas an exponential decay is obtained for the insulating solution obtained within the two-site DCA.

The much higher value of the critical interaction strength in the single-site approximation is due to the complete neglect of intersite correlations (here, mainly antiferromagnetic). These are known to have a crucial effect on the critical interaction strength, and the two-site approximation accounts for this. Furthermore, for example, Ref. [66] shows that the critical interaction strength of the two-site approximation is much closer to those of the
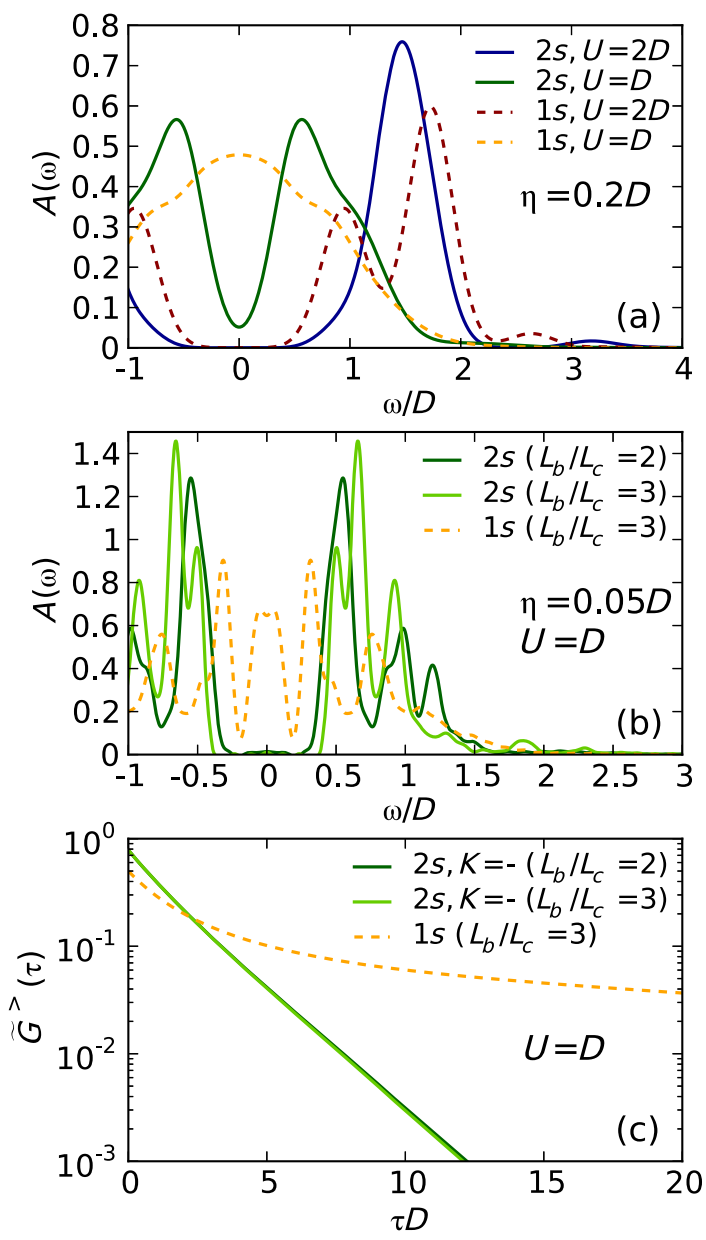

FIG. 6. Comparison of results obtained using imaginary-time MPS with $L_{b} / L_{c}=3$ for single-site ( $\left.1 s\right)$ and two-site (2s) DMFT approximations to the Hubbard-Kanamori model [Eq. (14)] on the two-dimensional square lattice with half-bandwidth $D=4 t$, $\varepsilon_{k}^{a b}=-2 t\left(\cos k_{x}+\cos k_{y}\right) \delta^{a b}, U^{\prime}=U-2 J, J=U / 4$, and $n=$ $3(\mu=5 U / 2-5 J)$, that is, in the particle-hole symmetric case. (a) Spectral functions for broadening $\eta=0.2 D$; (b) broadening $\eta=0.05 D$. In (c), we show the imaginary-time evolution of $\tilde{G}^{>}(\tau)$ as defined in Eq. (4a), confirming by comparison to a calculation for a smaller bath $L_{b} / L_{c}=2$ that this quantity has been converged with respect to the bath size. The maximal bond dimension for the ground state search is $m=1000$.

four- and eight-site approximations than to the single-site approximation.

\section{CONCLUSION}

This paper introduces an imaginary-time MPS (ITMPS) solver for DMFT and shows that it can treat complex models, not easily accessible with other methods, at modest computational cost. This development establishes DMRG as a flexible low-cost impurity solver for realistic problems, such as those encountered in the study of strongly correlated materials. The crucial advance stems from the fact that imaginary-time evolution does not create entanglement, and 
hence allows one to compute Green's functions numerically exactly, provided a ground state calculation is feasible. We note that this reduces the computational cost to the same level as necessary for the density matrix embedding theory [20], although the latter requires a smaller bath due to a different self-consistency condition.

The method can be improved in many ways. In particular, different representations of the impurity problem exhibit different degrees of entanglement, so optimizing the representation of the impurity problem is a promising route. Ideas from ED approaches for constructing relevant subspaces [21-24] of the Hilbert space may lead to further improvements. Such techniques have been successfully combined with MPS [67]. Another route to reduce computational effort and by that reach even more complex models could consist in performing computations for the reduced dynamics of the impurity [68]. Extending the method towards nonzero temperature requires the calculation of Green's functions at nonzero temperature, for which numerous MPS-based techniques have already been developed. At very low temperatures one could combine a strategy based on Lanczos algorithms [10] with its MPS implementation $[43,48]$ or use minimally entangled typical thermal states [69]. The most frequently used method is purification [70,71], whose usefulness for the calculation of spectral functions, combined with linear prediction, has been amply demonstrated [54,72-74]. At very high temperatures, an extremely cheap algorithm for computing Green's functions has been presented in Ref. [50]. Finally, we note that using MPS as an impurity solver makes using entanglement as a quantity for understanding the properties of the embedded impurity cluster very easily accessible. Proposals in this direction have been made for cellular DMFT [75] and for impurity models generally [76].

\section{ACKNOWLEDGMENTS}

F. A. W thanks G. K.-L. Chan for stressing the relevance of converging the DMFT loop on the imaginary-frequency axis, N.-O. Linden for helpful discussions, and Columbia University for hospitality during the main phase of this work. A. J. M. and U.S. acknowledge the hospitality of the Aspen Center for Physics NSF Grant No. 1066293 during the inception of this work. F. A. W. and U.S. acknowledge funding by FOR 1807 of the DFG. A. J. M. and A. G. were supported by the U.S. Department of Energy under Grant No. ER-046169.

\section{APPENDIX A: FURTHER TECHNICAL DETAILS}

\section{Ground state optimization}

The main challenge in solving the ground state problem of a typical cluster-bath Hamiltonian as encountered in DMFT stems from the fact that DMRG is a variational procedure that is initialized with a random state, which is then optimized locally. A local optimization procedure is slow when optimizing a global energy landscape. In addition, the local optimization is prone to getting stuck in local minima, if no "perturbation steps that mix symmetry sectors" are applied. The standard perturbation techniques for single-site DMRG [77-79] rely on "perturbation terms" that are produced by contracting the Hamiltonian with the MPS. If the Hamiltonian itself does not contain terms that mix the symmetry sector, these methods do not work.

A typical cluster-bath Hamiltonian has both features, a global variation of the potential energy and parts that are not connected with symmetry-mixing terms, such as in the three-band Hubbard-Kanamori model at $J=0$. This situation is sketched in Fig. 7.

In Ref. [39], the models under study allowed one to solve this problem using the noninteracting solution. For the general models studied in the present paper, an unbiased numerical technique has to be employed. What we do in practice is to first find the ground state of a system with additional symmetry-mixing couplings (denoted as red solid lines in Fig. 7) that are then adiabatically switched off. In practice, we sweep 5-10 times with additional hoppings of $10 \%$ magnitude of the physical hoppings, and another 5-10 times with additional hoppings of $1 \%$ magnitude. After these preliminary sweeps, the quantum number (e.g., particle number) distribution has globally converged, and we can continue with converging the ground state of the exact Hamiltonian.

\section{Convergence of DMFT iteration}

The calculations for the three-band single-site DMFT in Sec. IVA are only trivially parallelized using one core to compute the imaginary time evolution of each the particle $(>)$ and the hole $(<)$ Green functions $\tilde{G}^{\gtrless}(\tau)$.

In Fig. 8, we show the converged DMFT loop for the single-site DMFT for the three-band Hubbard-Kanamori model as studied in Fig. 5. Figure 8(a) shows the convergence of the Matsubara Green function down to a precision of $10^{-3}$. Figures $8(\mathrm{~b})$ and $8(\mathrm{c})$ show the convergence of the density and of the ground state energy per particle, respectively. Figure 8(d) shows the

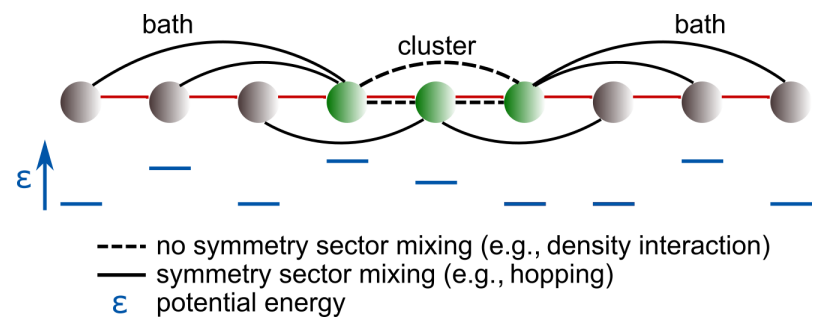

FIG. 7. Sketch of a typical cluster-bath Hamiltonian $\left(L_{c}=3\right.$, $L_{b}=6$ ) when it is mapped to a one-dimensional chain. Dashed lines depict couplings that do not mix symmetry sectors, and solid lines depict couplings that mix symmetry sectors. 

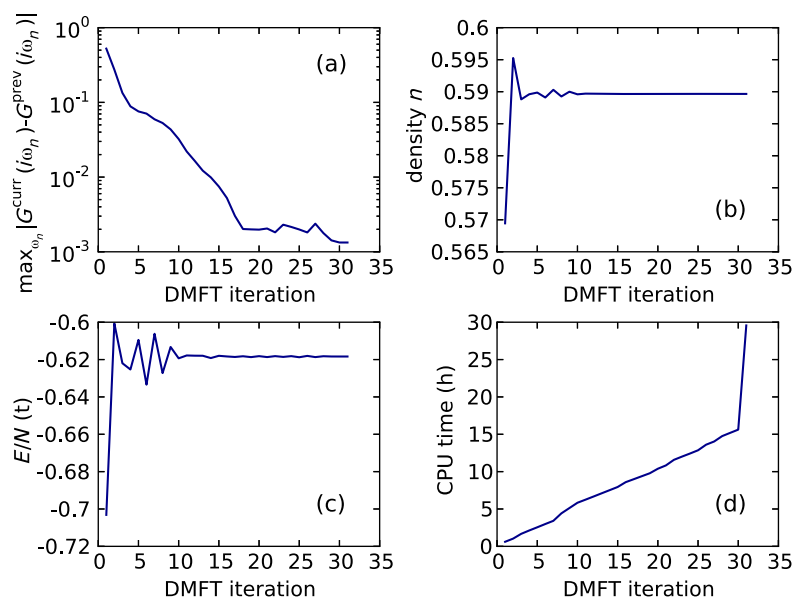

FIG. 8. Single-site DMFT for three-band Hubbard-Kanamori model as studied in Fig. 5. Here for the case $n=1.77(\mu=5.0)$ and $L_{b} / L_{c}=3$. To obtain the solution for $n=1.79$ as shown in Fig. 5, we choose $\mu=5.1$ and start from the $n=1.77$ solution. (a) Convergence of Matsubara Green's function in the DMFT loop, starting from the noninteracting solution. (b) Convergence of the density in the DMFT loop. (c) Convergence of the ground state energy per particle in the DMFT loop. (d) Computation time. An iteration on the Matsubara axis takes about $30 \mathrm{~min}$. The final real-axis computation (iteration 31 ) is considerably more expensive, but can still be optimized.

computation time. An iteration on the Matsubara axis takes about $30 \mathrm{~min}$. The final real-axis computation (iteration 31 ) is considerably more expensive, but can still be optimized.

\section{APPENDIX B: LEAST-ENTANGLED REPRESENTATION AND OFF-DIAGONAL HYBRIDIZATION FUNCTIONS}

\section{Geometry and general considerations}

In Ref. [40], some of us showed that the star geometry of the impurity problem can have substantially lower entanglement than its chain geometry. In the star geometry, DMRG profits from the small entanglement of the almost occupied states with low potential energy with the almost unoccupied states with high potential energy. A high weight for the superposition of a low- with a high-energy state is physically irrelevant. In the star geometry, DMRG is able to eliminate these superpositions as potential energy is separated locally, i.e., in the same basis in which DMRG optimizes the reduced density matrix in order to discard irrelevant contributions. In principle, as mentioned in Appendix C, ideas from basis-selective approaches in exact diagonalization are a different method to account for the fact that many states in the Hilbert space have a negligible weight for the computation of the Green function and only few physically relevant states occupy a small fraction of the Hilbert space. Among these are the truncated configuration interaction $[20,23,24,56]$, the basis-selective ED [21], or the coupled cluster methods in quantum chemistry. As these methods can be combined with DMRG [67], they might be a further route to construct efficient representations of the impurity-cluster problem.

In the present paper, the question of the least entangled representation of the impurity problem is restricted to the question of which basis to choose in a DCA calculation. This is of high relevance also in another context: In the realspace representation, the hybridization function becomes off-diagonal. For the CTQMC method, this generates a sign problem. In our approach, this does not affect computational cost much in the single-band Hubbard model. It even leads to a tremendous reduction of computational cost for the three-band Hubbard-Kanamori interaction.

\section{DCA in momentum or real space}

The complexity of the interaction determines whether the real- or the momentum-space representation of the clusterbath Hamiltonian is less entangled. In real space, the interaction has a simple form, but the hybridization function has off-diagonal contributions, which result in additional couplings of cluster and bath sites. In momentum space, the hybridization function is diagonal but the interaction becomes off diagonal. The additional couplings induced by that depend on the complexity of the interaction.

Let us be more concrete. For the two-site case, the discrete Fourier transform yields the even and odd superposition of the real-space cluster.

$$
\begin{aligned}
& \tilde{d}_{1}^{\dagger}=\frac{1}{\sqrt{2}}\left(d_{1}^{\dagger}+d_{2}^{\dagger}\right), \\
& \tilde{d}_{2}^{\dagger}=\frac{1}{\sqrt{2}}\left(d_{1}^{\dagger}-d_{2}^{\dagger}\right),
\end{aligned}
$$

where the index of $\tilde{d}_{K}^{\dagger}$ labels momentum patches $K$ and the index of $d_{i}^{\dagger}$ labels real-space cluster sites $i$. There might be further indices labeling spin or orbital.

In real space, the hybridization function has the form

$$
\Lambda_{i j}(z)=\sum_{l=1}^{L_{b}} \frac{V_{i l}^{*} V_{j l}}{z-\varepsilon_{l}},
$$

where the symmetry of the real-space cluster imposes $\Lambda_{i j}(z)=\Lambda_{j i}(z)$. In momentum space, the hybridization function is diagonal,

$$
\tilde{\Lambda}_{K}(z)=\sum_{l=l_{K}=1}^{L_{b}^{\prime}} \frac{\tilde{V}_{K l}^{*} \tilde{V}_{K l}}{z-\tilde{\varepsilon}_{K l}},
$$

and symmetry is reflected in the reduced number of bath sites per patch, $L_{b}^{\prime}=L_{b} / L_{c}$, where $L_{c}=2$ is the number of momentum patches. 
We choose to use the momentum representation for the bath discretization, as was done for the real axis in Ref. [39]. While on the real-frequency axis this is the only viable option, the bath fitting on the imaginary-frequency axis via Eq. (13) is possible also for the off-diagonal realspace case. In real space, e.g., particle-hole symmetry can be easily imposed in the fitting procedure, while this is not possible in momentum space.

Given the parameters of the momentum-space representation obtained by performing a bath fit via Eq. (13), we define the parameters of the equivalent real-space representation as follows: In momentum space, bath parameters are indexed by $l_{K}=1, \ldots, L_{b}^{\prime}, L_{b}^{\prime}=L_{b} / L_{c}$, and in real space, bath parameters are indexed by $l=1, \ldots, L_{b}$, then

$$
\begin{aligned}
\varepsilon_{l} & =\tilde{\varepsilon}_{1, l_{1}=l}, \quad \text { for } l=1, \ldots, L_{b}^{\prime}, \\
\varepsilon_{l} & =\tilde{\varepsilon}_{2, l_{2}=l-L_{b}^{\prime}}, \quad \text { for } l=L_{b}^{\prime}+1, \ldots, L_{b}, \\
V_{1 l} & =V_{2 l}=\frac{1}{\sqrt{2}} \tilde{V}_{1, l_{1}=l}, \quad \text { for } l=1, \ldots, L_{b}^{\prime}, \\
V_{1 l} & =-V_{2 l}=\frac{1}{\sqrt{2}} \tilde{V}_{2, l_{2}=l-L_{b}^{\prime}} \text { for } l=L_{b}^{\prime}+1, \ldots, L_{b} .
\end{aligned}
$$

Whereas the momentum-space Hamiltonian has $L_{b}$ nonzero couplings $V_{K l_{K}}$, the real-space Hamiltonian has $L_{c} \times L_{b}$ couplings $V_{i l}$. On the other hand, the interaction part generates $L_{c} \times\left(L_{c}-1\right)$ additional nonlocal couplings in the momentum-space representation as compared to the real-space Hamiltonian.

From this one could naively expect that the real-space representation is less entangled if $L_{c} \times\left(L_{c}-1\right)>L_{c} \times L_{b}$. Numerical experiments show that the real-space representation is much more favorable than this estimate. For a single-band Hubbard model, we find about the same entanglement in the real-space and the momentum-space representation, with slight advantages for momentum space. In the three-band Hubbard-Kanamori model, the real-space representation is considerably less entangled and leads to a tremendous reduction of computational cost. In particular, we are not able to obtain the results of Fig. 6 in the momentum-space representation when using $L_{b} / L_{c}=3$, only for $L_{b} / L_{c}=2$ but then at much higher computational cost.

\section{APPENDIX C: GREEN'S FUNCTIONS FROM MATRIX PRODUCT STATES}

Even though the following discussion is not needed to set up the imaginary-time MPS impurity solver, it describes the context of the method.

A computation of $A(\omega)=\left\langle\psi_{0}\left|\delta\left(\omega-\left(H-E_{0}\right)\right)\right| \psi_{0}\right\rangle$ via a computation of all eigenstates of $H$ is extremely redundant as only a tiny neighborhood $\mathcal{N}=\left\{|\psi\rangle \mid\left\langle\psi|H| \psi_{0}\right\rangle \neq 0\right\}$ of a the single-particle excitation $\left|\psi_{0}\right\rangle$ contributes in the sum (inserting identities $\left.\sum_{n}\left|E_{n}\right\rangle\left\langle E_{n}\right|\right)$ in $A(\omega)$. In ED, this is exploited by systematically constructing the subspace $\mathcal{N}$ by spanning it using particle-hole excitations [20,21], which might also be a viable route for further developments within DMRG [67]. In DMRG, one needs to make a statement about the entanglement of the states in the subspace $\mathcal{N}$ : one might note that these are, in general, more strongly entangled than the single-particle excitation $\left|\psi_{0}\right\rangle$, but should still be much less entangled than the rest of the Hilbert space. This is illustrated in Fig. 9.

In Ref. [30], some of us argued that expanding the spectral function in a family of orthogonal functions is a natural way to construct a basis for $\mathcal{N}$, starting from the lowly entangled $\left|\psi_{0}\right\rangle$ and successively increasing entanglement of states and thereby computational complexity in a sequence of basis states $\left|\psi_{n}\right\rangle$. Reference [30] discussed the expansion of $A(\omega)$ in Chebyshev polynomials $T_{n}(\omega / a)=\arccos [n \cos (\omega / a)]$, which are orthogonal with respect to an inner product weighted by $w(x)=\sqrt{1-x^{2}}$ [80], and in the plane waves $\exp [i \omega(n / a)]$ [orthogonal with weight function $w(x)=1$ ], where the energy $a$ is chosen larger than the support of $A(\omega)$. The associated generated sequences of basis states are then

$$
\begin{aligned}
\left|\psi_{n}^{\text {Che }}\right\rangle & =2\left(\frac{H-E_{0}}{a}+b\right)\left|\psi_{n-1}^{\text {Che }}\right\rangle-\left|\psi_{n-2}^{\text {Che }}\right\rangle, \\
\left|\psi_{n}^{\text {time }}\right\rangle & =\exp \left[-i\left(H-E_{0}\right) \frac{n}{a}\right]\left|\psi_{0}\right\rangle,
\end{aligned}
$$

and have different entanglement properties. The states $\left|\psi_{n}^{\text {time }}\right\rangle$ associated with time evolution are in general less entangled than the states $\left|\psi_{n}^{\text {Che }}\right\rangle$ associated with the Chebyshev recursion [30]. This is due to the observation that error accumulation in the Chebyshev recursion is worse conditioned than in time propagation [30], which necessitates keeping the error in a single step of the Chebyshev recursion much smaller than in the equivalent

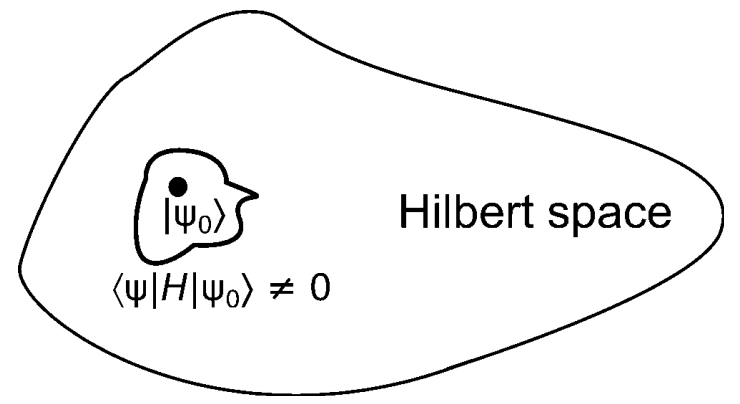

FIG. 9. Single-particle excitation $\left|\psi_{0}\right\rangle$ of the ground state $\left|E_{0}\right\rangle$ and the subspace $\mathcal{N}=\left\{|\psi\rangle \mid\left\langle\psi|H| \psi_{0}\right\rangle \neq 0\right\}$ of the Hilbert space that is relevant for the computation of a single-particle spectral function of the form $\left\langle\psi_{0}|\delta(\omega-H)| \psi_{0}\right\rangle$. The single-particle excitation is very lowly entangled, the subspace is more strongly entangled, but still in general more lowly entangled than the rest of the Hilbert space. 
time-evolution step, which in turn requires the use of higher bond dimensions in the Chebyshev recursion making it less efficient. In addition to the statements of Ref. [30], we note here that the sequence produced by the Lanczos algorithm,

$\left|\psi_{n}^{\mathrm{Lan}}\right\rangle=H-\alpha_{n}\left|\psi_{n-1}^{\mathrm{Lan}}\right\rangle-\left|\psi_{n-2}^{\mathrm{Lan}}\right\rangle, \quad \alpha_{n}, \quad \beta_{n} \in \mathbb{R}$,

can be associated with an expansion of the spectral function in polynomials that are orthogonal with respect to an inner product weighted by $w(x)=A(x)$ [81]. This is very efficient but numerically unstable.

In contrast to the previous methods, which generate an increasingly complex basis when determining the spectral function to a higher and higher precision, correction-vector DMRG aims to optimize a state in frequency space, which a priori contains contributions that have undergone an infinitely long time evolution. As time evolution creates entanglement, these states are much too strongly entangled for an efficient treatment. They are "far away" from the controlled, lowly entangled single-particle excitation $\left|\psi_{0}\right\rangle$. In order to still perform a meaningful computation in frequency space, one introduces a so-called (Lorentzian) broadening parameter $\eta$ that damps out contributions from an infinite time evolution. One then does not obtain the exact spectral function but a broadened version as in Eq. (10). The broadening parameter has to be guessed a priori: If it is chosen too small, high entanglement prevents convergence of the calculation. If it is chosen too large, one will be far from the exact version of the spectral function. In the expansion methods discussed above, by contrast, one can stop the computation simply when it becomes too costly. If one has not recovered the exact $A(\omega)$ at this point, a broadened version can be systematically constructed with an a posteriori determined $\eta$ as in Eq. (10).

[1] W. Metzner and D. Vollhardt, Correlated Lattice Fermions in $d=\infty$ Dimensions, Phys. Rev. Lett. 62, 324 (1989).

[2] A. Georges and G. Kotliar, Hubbard Model in Infinite Dimensions, Phys. Rev. B 45, 6479 (1992).

[3] A. Georges, G. Kotliar, W. Krauth, and M. J. Rozenberg, Dynamical Mean-Field Theory of Strongly Correlated Fermion Systems and the Limit of Infinite Dimensions, Rev. Mod. Phys. 68, 13 (1996).

[4] T. Maier, M. Jarrell, T. Pruschke, and M. Hettler, Quantum Cluster Theories, Rev. Mod. Phys. 77, 1027 (2005).

[5] G. Kotliar, S. Savrasov, K. Haule, V. Oudovenko, O. Parcollet, and C. Marianetti, Electronic Structure Calculations with Dynamical Mean-Field Theory, Rev. Mod. Phys. 78, 865 (2006).

[6] A. N. Rubtsov, V. V. Savkin, and A. I. Lichtenstein, Continuous-Time Quantum Monte Carlo Method for Fermions, Phys. Rev. B 72, 035122 (2005).
[7] P. Werner, A. Comanac, L. de Medici, M. Troyer, and A. J. Millis, A Continuous-Time Solver for Quantum Impurity Models, Phys. Rev. Lett. 97, 076405 (2006).

[8] E. Gull, A. J. Millis, A. I. Lichtenstein, A. N. Rubtsov, M. Troyer, and P. Werner, Continuous-Time Monte Carlo Methods for Quantum Impurity Models, Rev. Mod. Phys. 83, 349 (2011).

[9] M. Caffarel and W. Krauth, Exact Diagonalization Approach to Correlated Fermions in Infinite Dimensions: Mott Transition and Superconductivity, Phys. Rev. Lett. 72, 1545 (1994).

[10] M. Capone, L. de Medici, and A. Georges, Solving the Dynamical Mean-Field Theory at Very Low Temperatures Using the Lanczos Exact Diagonalization, Phys. Rev. B 76, 245116 (2007).

[11] A. Liebsch and H. Ishida, Temperature and Bath Size in Exact Diagonalization Dynamical Mean Field Theory, J. Phys. Condens. Matter 24, 053201 (2012).

[12] R. Bulla, T. Costi, and T. Pruschke, Numerical Renormalization Group Method for Quantum Impurity Systems, Rev. Mod. Phys. 80, 395 (2008).

[13] D. J. García, K. Hallberg, and M. J. Rozenberg, Dynamical Mean Field Theory with the Density Matrix Renormalization Group, Phys. Rev. Lett. 93, 246403 (2004).

[14] H. Li and N.-H. Tong, A Standard Basis Operator Equation of Motion Impurity Solver for Dynamical Mean Field Theory, arXiv:1501.07689.

[15] P. Wang, G. Cohen, and S. Xu, Numerical Operator Method for the Real-Time Dynamics of Strongly Correlated Quantum Impurity Systems Far from Equilibrium, Phys. Rev. B 91, 155148 (2015).

[16] L.-F. Arsenault, O. A. von Lilienfeld, and A. J. Millis, Machine Learning for Many-Body Physics: Efficient Solution of Dynamical Mean-Field Theory, arXiv:1506.08858.

[17] M. Schüler, C. Renk, and T. O. Wehling, Variational Exact Diagonalization Method for Anderson Impurity Models, Phys. Rev. B 91, 235142 (2015).

[18] M. Granath and H. U.R. Strand, Distributional Exact Diagonalization Formalism for Quantum Impurity Models, Phys. Rev. B 86, 115111 (2012).

[19] H. Shinaoka, M. Dolfi, M. Troyer, and P. Werner, Hybridization Expansion Monte Carlo Simulation of Multi-Orbital Quantum Impurity Problems: Matrix Product Formalism and Improved Monte Carlo Sampling, J. Stat. Mech. (2014) P06012.

[20] G. Knizia and G. K.-L. Chan, Density Matrix Embedding: A Simple Alternative to Dynamical Mean-Field Theory, Phys. Rev. Lett. 109, 186404 (2012).

[21] Y. Lu, M. Höppner, O. Gunnarsson, and M. W. Haverkort, Efficient Real-Frequency Solver for Dynamical Mean-Field Theory, Phys. Rev. B 90, 085102 (2014).

[22] D. Zgid, E. Gull, and G. Chan, Truncated Configuration Interaction Expansions as Solvers for Correlated Quantum Impurity Models and Dynamical Mean Field Theory, Phys. Rev. B 86, 165128 (2012).

[23] C. Lin and A. A. Demkov, Efficient Variational Approach to the Impurity Problem and Its Application to the Dynamical Mean-Field Theory, Phys. Rev. B 88, 035123 (2013).

[24] C. Lin and A. A. Demkov, Electron Correlation in Oxygen Vacancy in $\mathrm{SrTiO}_{3}$, Phys. Rev. Lett. 111, 217601 (2013). 
[25] K. M. Stadler, A. Weichselbaum, Z. P. Yin, J. von Delft, and G. Kotliar, DMFT + NRG Study of Spin-Orbital Separation in a Three-Band Hund's Metal, Phys. Rev. Lett. 115, 136401 (2015).

[26] S. R. White, Density Matrix Formulation for Quantum Renormalization Groups, Phys. Rev. Lett. 69, 2863 (1992).

[27] U. Schollwöck, The Density-Matrix Renormalization Group in the Age of Matrix Product States, Ann. Phys. (Amsterdam) 326, 96 (2011).

[28] U. Schollwöck, The Density-Matrix Renormalization Group, Rev. Mod. Phys. 77, 259 (2005).

[29] A. Holzner, A. Weichselbaum, I. P. McCulloch, U. Schollwöck, and J. von Delft, Chebyshev Matrix Product State Approach for Spectral Functions, Phys. Rev. B 83, 195115 (2011).

[30] F. A. Wolf, J. A. Justiniano, I. P. McCulloch, and U. Schollwöck, Spectral Functions and Time Evolution from the Chebyshev Recursion, Phys. Rev. B 91, 115144 (2015).

[31] S. Nishimoto, F. Gebhard, and E. Jeckelmann, Dynamical Density-Matrix Renormalization Group for the MottHubbard Insulator in High Dimensions, J. Phys. Condens. Matter 16, 7063 (2004).

[32] M. Karski, C. Raas, and G. S. Uhrig, Electron Spectra Close to a Metal-to-Insulator Transition, Phys. Rev. B 72, 113110 (2005).

[33] S. Nishimoto, F. Gebhard, and E. Jeckelmann, Dynamical Mean-Field Theory Calculation with the Dynamical DensityMatrix Renormalization Group, Physica (Amsterdam) 378-380B, 283 (2006).

[34] D. J. García, E. Miranda, K. Hallberg, and M. J. Rozenberg, Mott Transition in the Hubbard Model Away from ParticleHole Symmetry, Phys. Rev. B 75, 121102 (2007).

[35] M. Karski, C. Raas, and G. S. Uhrig, Single-Particle Dynamics in the Vicinity of the Mott-Hubbard Metal-toInsulator Transition, Phys. Rev. B 77, 075116 (2008).

[36] R. Peters, Spectral Functions for Single- and Multi-impurity Models Using Density Matrix Renormalization Group, Phys. Rev. B 84, 075139 (2011).

[37] M. Ganahl, P. Thunström, F. Verstraete, K. Held, and H. G. Evertz, Chebyshev Expansion for Impurity Models Using Matrix Product States, Phys. Rev. B 90, 045144 (2014).

[38] M. Ganahl, M. Aichhorn, P. Thunström, K. Held, H. G. Evertz, and F. Verstraete, Efficient DMFT Impurity Solver Using Real-Time Dynamics with Matrix Product States, Phys. Rev. B 92, 155132 (2015).

[39] F. A. Wolf, I. P. McCulloch, O. Parcollet, and U. Schollwöck, Chebyshev Matrix Product State Impurity Solver for Dynamical Mean-Field Theory, Phys. Rev. B 90, 115124 (2014).

[40] F. A. Wolf, I. P. McCulloch, and U. Schollwöck, Solving Nonequilibrium Dynamical Mean-Field Theory Using Matrix Product States, Phys. Rev. B 90, 235131 (2014).

[41] C. Gramsch, K. Balzer, M. Eckstein, and M. Kollar, Hamiltonian-Based Impurity Solver for Nonequilibrium Dynamical Mean-Field Theory, Phys. Rev. B 88, 235106 (2013).

[42] K. Balzer, F. A. Wolf, I. P. McCulloch, P. Werner, and M. Eckstein, Nonthermal Melting of Néel Order in the Hubbard Model, Phys. Rev. X 5, 031039 (2015).
[43] K. A. Hallberg, Density-Matrix Algorithm for the Calculation of Dynamical Properties of Low-Dimensional Systems, Phys. Rev. B 52, R9827 (1995).

[44] T. D. Kühner and S. R. White, Dynamical Correlation Functions Using the Density Matrix Renormalization Group, Phys. Rev. B 60, 335 (1999).

[45] E. Jeckelmann, Dynamical Density-Matrix Renormalization-Group Method, Phys. Rev. B 66, 045114 (2002).

[46] S. R. White and A. E. Feiguin, Real-Time Evolution Using the Density Matrix Renormalization Group, Phys. Rev. Lett. 93, 076401 (2004).

[47] S. R. White and I. Affleck, Spectral Function for the $S=1$ Heisenberg Antiferromagetic Chain, Phys. Rev. B 77, 134437 (2008).

[48] P. E. Dargel, A. Wöllert, A. Honecker, I. P. McCulloch, U. Schollwöck, and T. Pruschke, Lanczos Algorithm with Matrix Product States for Dynamical Correlation Functions, Phys. Rev. B 85, 205119 (2012).

[49] A. Braun and P. Schmitteckert, Numerical Evaluation of Green's Functions Based on the Chebyshev Expansion, Phys. Rev. B 90, 165112 (2014).

[50] D. V. Savostyanov, S. V. Dolgov, J. M. Werner, and I. Kuprov, Exact NMR Simulation of Protein-Size Spin Systems Using Tensor Train Formalism, Phys. Rev. B 90, 085139 (2014).

[51] M. Hochbruck and C. Lubich, On Krylov Subspace Approximations to the Matrix Exponential Operator, SIAM J. Numer. Anal. 34, 1911 (1997).

[52] M. Ferrero, P. S. Cornaglia, L. De Leo, O. Parcollet, G. Kotliar, and A. Georges, Pseudogap Opening and Formation of Fermi Arcs as an Orbital-Selective Mott Transition in Momentum Space, Phys. Rev. B 80, 064501 (2009).

[53] W. H. Press, S. A. Teukolsky, W. T. Vetterling, and B. P. Flannery, Numerical Recipes: The Art of Scientific Computing, 3rd ed. (Cambridge University Press, New York, 2007).

[54] T. Barthel, U. Schollwöck, and S. R. White, Spectral Functions in One-Dimensional Quantum Systems at Finite Temperature Using the Density Matrix Renormalization Group, Phys. Rev. B 79, 245101 (2009).

[55] I. de Vega, U. Schollwöck, and F. A. Wolf, How to Discretize a Quantum Bath for Real-Time Evolution, Phys. Rev. B 92, 155126 (2015).

[56] A. Go and A. J. Millis, Spatial Correlations and the Insulating Phase of the High- $T_{c}$ Cuprates: Insights from a Configuration-Interaction-Based Solver for Dynamical Mean Field Theory, Phys. Rev. Lett. 114, 016402 (2015).

[57] D. Senechal, Bath Optimization in the Cellular Dynamical Mean-Field Theory, Phys. Rev. B 81, 235125 (2010).

[58] A. Dorda, M. Nuss, W. von der Linden, and E. Arrigoni, Auxiliary Master Equation Approach to Nonequilibrium Correlated Impurities, Phys. Rev. B 89, 165105 (2014).

[59] D. J. Wales and J.P. K. Doye, Global Optimization by Basin-Hopping and the Lowest Energy Structures of Lennard-Jones Clusters Containing Up to 110 Atoms, J. Phys. Chem. A 101, 5111 (1997).

[60] A. Georges, L. de' Medici, and J. Mravlje, Strong Electronic Correlations from Hund's Coupling, Annu. Rev. Condens. Matter Phys. 4, 137 (2013). 
[61] P. Werner, E. Gull, and A. J. Millis, Metal-Insulator Phase Diagram and Orbital Selectivity in Three-Orbital Models with Rotationally Invariant Hund Coupling, Phys. Rev. B 79, 115119 (2009).

[62] P. Werner, E. Gull, M. Troyer, and A. J. Millis, Spin Freezing Transition and Non-Fermi-Liquid Self-Energy in a ThreeOrbital Model, Phys. Rev. Lett. 101, 166405 (2008).

[63] Y. Nomura, S. Sakai, and R. Arita, Multiorbital Cluster Dynamical Mean-Field Theory with an Improved Continuous-Time Quantum Monte Carlo Algorithm, Phys. Rev. B 89, 195146 (2014).

[64] Y. Nomura, S. Sakai, and R. Arita, Nonlocal Correlations Induced by Hund's Coupling: A Cluster DMFT Study, Phys. Rev. B 91, 235107 (2015).

[65] L. de' Medici, J. Mravlje, and A. Georges, Janus-Faced Influence of the Hund's Rule Coupling in Strongly Correlated Materials, Phys. Rev. Lett. 107, 256401 (2011).

[66] E. Gull, M. Ferrero, O. Parcollet, A. Georges, and A. J. Millis, Momentum-Space Anisotropy and Pseudogaps: A Comparative Cluster Dynamical Mean-Field Analysis of the Doping-Driven Metal-Insulator Transition in the TwoDimensional Hubbard Model, Phys. Rev. B 82, 155101 (2010).

[67] Y. Ma and H. Ma, Assessment of Various Natural Orbitals as the Basis of Large Active Space Density Matrix Renormalization Group Calculations, J. Chem. Phys. 138, 224105 (2013).

[68] G. Cohen, E. Y. Wilner, and E. Rabani, Generalized Projected Dynamics for Non-System Observables of Non-Equilibrium Quantum Impurity Models, New J. Phys. 15, 073018 (2013).

[69] S. R. White, Minimally Entangled Typical Quantum States at Finite Temperature, Phys. Rev. Lett. 102, 190601 (2009).

[70] F. Verstraete, J. J. Garcia-Ripoll, and J. I. Cirac, Matrix Product Density Operators: Simulation of Finite-
Temperature and Dissipative Systems, Phys. Rev. Lett. 93, 207204 (2004).

[71] M. Zwolak and G. Vidal, Mixed-State Dynamics in OneDimensional Quantum Lattice Systems: A Time-Dependent Superoperator Renormalization Algorithm, Phys. Rev. Lett. 93, 207205 (2004).

[72] C. Karrasch, J. H. Bardarson, and J. E. Moore, Finite Temperature Dynamical Density Matrix Renormalization Group and the Drude Weight of Spin-1/2 Chains, Phys. Rev. Lett. 108, 227206 (2012).

[73] T. Barthel, Precise Evaluation of Thermal Response Functions by Optimized Density Matrix Renormalization Group Schemes, New J. Phys. 15, 073010 (2013).

[74] B. Lake, D. A. Tennant, J. S. Caux, T. Barthel, U. Schollwöck, S. E. Nagler, and C. D. Frost, Multispinon Continua at Zero and Finite Temperature in a Near-Ideal Heisenberg Chain, Phys. Rev. Lett. 111, 137205 (2013).

[75] M. Udagawa and Y. Motome, Entanglement Spectrum in Cluster Dynamical Mean-Field Theory, J. Stat. Mech. (2015) P01016.

[76] S. S. B. Lee, J. Park, and H. S. Sim, Macroscopic Quantum Entanglement of a Kondo Cloud at Finite Temperature, Phys. Rev. Lett. 114, 057203 (2015).

[77] S. R. White, Density Matrix Renormalization Group Algorithms with a Single Center Site, Phys. Rev. B 72, 180403 (2005).

[78] S. V. Dolgov and D. V. Savostyanov, Alternating Minimal Energy Methods for Linear Systems in Higher Dimensions, SIAM J. Sci. Comput. 36, A2248 (2014).

[79] C. Hubig, I. P. McCulloch, U. Schollwöck, and F. A. Wolf, Strictly Single-Site DMRG Algorithm with Subspace Expansion, Phys. Rev. B 91, 155115 (2015).

[80] A. Weiße, G. Wellein, A. Alvermann, and H. Fehske, The Kernel Polynomial Method, Rev. Mod. Phys. 78, 275 (2006).

[81] W. Gautschi, Orthogonal Polynomials (in Matlab), J. Comput. Appl. Math. 178, 215 (2005). 